\title{
Error fields in the Wendelstein 7-X stellarator
}

\author{
Samuel A. Lazerson \\ Princeton Plasma Physics Laboratory, Princeton, NJ \\ E-mail: lazerson@pppl.gov \\ Sergey Bozhenkov \\ Max-Planck Institut für Plasmaphysik, Greifswald, Germany
}

\section{Ben Israeli}

Princeton Plasma Physics Laboratory, Princeton, NJ

\author{
Matthias Otte, Holger Niemann, Victor Bykov, Michael \\ Endler, Tamara Andreeva, Adnan Ali, Peter Drewelow, Marcin \\ Jakubowski, Aleix Puig Sitjes \\ Max-Planck Institut für Plasmaphysik, Greifswald, Germany
}

\section{Fabio Pisano, Barbara Cannas}

University of Cagliari, Cagliari, Italy

\section{and the W7-X Team}

\begin{abstract}
The role of error fields in stellarators is explored in this work through simulations of and experiments performed on the Wendelstein 7-X (W7-X) stellarator. Previous experimental results, based on limiter plasmas in W7-X, suggest that error fields are small in the experiment based on limiter plasmas. In this work, we document results from the first divertor campaign on W7-X with a focus on error fields resonant with the edge island divertor structure. Divertor thermocouples measurements corroborate earlier limiter results that the error fields are small and correctible, requiring only $5 \%$ the total rated current of the trim coil system. The effect of these error fields on magnetic geometry and topology are investigated using the technique of flux surface measurement. The resulting divertor thermal load asymmetry is modeled via field line diffusion and compared to experimental data.

$\dagger$ Notice: This manuscript has been authored by Princeton University under Contract Number DEAC02-09CH11466 with the U.S. Department of Energy. The publisher, by accepting the article for publication acknowledges, that the United States Government retains a non-exclusive, paid-up, irrevocable, world-wide license to publish or reproduce the published form of this manuscript, or allow
\end{abstract} others to do so, for United States Government purposes. 
Keywords: Fusion, Magnetic Fields, Stellarator

Submitted to: Plasma Phys. Control. Fusion 


\section{Introduction}

The goal of magnetically confined nuclear fusion is to tailor magnetic fields to form flux surfaces which confine a hot plasma, thus the minimization of errors in the magnetic field structure are of manifest relevance [1]. This is especially true of stellarators, in which the confining magnetic field arrises predominantly from external electromagnetic coils. Stellarators confine plasmas through careful three dimensional shaping of the magnetic flux surfaces produced by these coils. Manufacturing defects can result in deformations of said flux surfaces, topological breaking of flux surfaces, and periodicity changes in the magnetic field, which all reduce confinement times. In this work we analyze experimental data obtained from the Wendelstein 7-X (W7-X) stellarator to better understand the role and influence of error fields in stellarators.

Before discussing W7-X, it is worthwhile to review the history of stellarators with a focus on the effect that error fields had on their performance. Beginning with one of the earliest stellarators (Model C) the effect of perturbing magnetic fields on flux surfaces were already being investigated. Here it was shown that addition of a vertical field could significantly degrade flux surface quality, and comparisons between measurements and computer models did show discrepancies which (by today's standards) would have suggested the presence of additional, unaccounted for magnetic fields [2]. The Advanced Toroidal Facility (ATF) used flux surface mapping techniques to measure the amplitude of a $n / m=1 / 2$ island. The source of this field was attributed to coil bus-work and was corrected [3]. The URAGAN-3 stellarator built on the ATF results to measure and correct error fields associated with misalignment of their vertical field coil pair [4]. The W7-AS stellarator performed extensive flux surface mapping of its configuration space finding both low and high order islands. Initial analysis could not conclude the source of these fields, here it was found that they did not scale with magnetic field strength, suggesting they arose from the coil system and current leads [5]. At the end of the experimental campaign, measurements were made of the edge island structure, confirming a change in rotational transform which was dependent on field strength. This was attributed to elastic deformations of the non-planar field coils [6]. The WEGA stellarator had large $n / m=1 / 3$ and $n / m=1 / 4$ island chains present and was able to image island chains with polodial mode numbers up to $m=9$ [7]. The error field was attributed to mis-alignments between the helix and toroidal field coils. An ability to control island size through an external magnetic coil was also demonstrated. The W7$\mathrm{X}$ flux surface mapping system capable of permanent installation and 2.5T operation was developed on WEGA. The Compact Toroidal Hybrid device found that a proper fix between modeled and measured axis position required a modified coil set based on measurements of the coil set [8]. The TJ-II device also measured error fields which were attributed to deviations in the toroidal field coils positions. These fields resulted in larger than expected islands at rational surfaces [9]. The desire to control error fields has even led to the cancelation of projects, as was the case for National Compact Stellarator Experiment (NCSX). Here mounting cost over-runs largely associated with 
coil tolerances ultimately ended the program before it could operate $[10,11]$. The Large Helical Device (LHD) observed both $n / m=1 / 1$ and $n / m=1 / 2$ island chains by flux surface mapping. These islands were attributed to ferromagnetic materials in the torus hall and shown to be correctible by the Local Island Divertor (LID) coils [12]. In the H1-NF heliac, flux surface measurments were matched to deformations of the coils models to produce an improved coil model. The improved coil model indicated strong changes in the magnetic well profile near the edge of the plasma. [13] In the Columbia Non-neutral Torus (CNT) flux surface measurements were used to directly calculate the displacements of the coils [14]. It was later confirmed through numerical simulations that these displacements were consistent with those most likely to influence plasma shape [15]. Finally, it should be noted that while we have focused on stellarators, tokamaks also suffers from similar effects of error fields. In these devices, error fields can significantly reduce performance and provide 'seed' fields for a variety of symmetry breaking instabilities [16]. The desire to minimize these effects has similarly contributed to increases in cost of the ITER project. Clearly, the study of these fields, their correction, and development of robust configurations is of manifest relevance to the entire nuclear fusion program.

The W7-X experiment serves as an excellent case-study in the effects of error fields, given its coil set [17, 18], diagnostic set [19], and reactor relevance. The W7-X device is a five field period quasi-isodynamic stellarator with a plasma volume of approximately 30 $m^{3}$. The three dimensional shape of the plasma arrises from a set of 50 non-planar and 20 planar superconducting magnetic coils. The shaping of the magnetic field was optimized to confine the thermal part of the plasma distribution which undergoes a bounce motion between maxima in magnetic field strength [20]. Unintended fields can disturb the shape of these flux surfaces reducing the effective confinement of such particles. The interaction of the plasma with the first wall structures is controlled through the presence of a four, five, or six lobed magnetic island chain $(\iota=5 / 4,5 / 5,5 / 6)$. Error fields which resonate with this magnetic structure have been predicted to result in overloading of first wall structures [21]. In particular magnetic fields with $n / m=1 / 1,2 / 2,3 / 3,4 / 4$ must be minimized. As a consequence, a great deal of effort has been spent to minimize said fields during construction [22]. This has yielded a device with low levels of resonant error [23].

It should be noted that finite plasma beta will also change the island position and structure, thereby changing the plasma wall interaction. These changes are nominally stellarator symmetric and preserve the five-fold symmetry of the device. However, they can amplify the effect of the error fields. In this work we focus on exploration of the error field effects themselves and leave a discussion of the interplay between plasma response and error fields to future work.

In the next section we will review the sources of error fields in the W7-X experiment and how they can be measured. In section 3, we will review experimental evidence of their effect on plasma operation. We will conclude in section 4 with a discussion of what can be done in future devices to tolerate, compensate, and minimize error fields. 

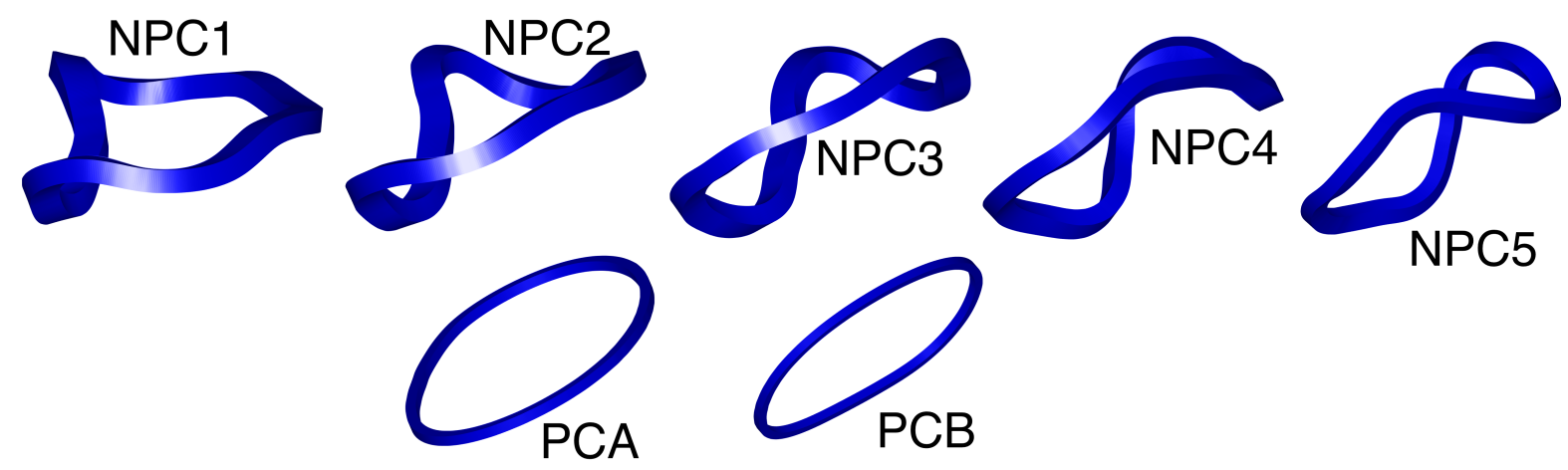

Figure 1. The seven superconducting coil types of the Wendelstein 7-X experiment. Each of five identical modules of W7-X possess a stellarator symmetry (flip mirror about the half module) so that these 7 coil types result in 70 coils in total.

\section{Sources of error fields}

Any unintended magnetic field can be labeled an error field, and thus arise from a multitude of sources. Deviations in the winding of magnetic coils, errors in the placement of coil relative to each other, electromagnetic deformation of the coils, current feeds, auxiliary coils, and magnetic material can all produce error fields. Error fields are ubiquitous and impossible to avoid. One may then conclude they are ultimately terminal to the endeavor of magnetically confined nuclear fusion. And while these points are true, two additional facts negate this terminal conclusion. The first of which is that not all errors in the magnetic field matter equally. Secondly, error fields can be compensated via additional auxiliary magnetic coils. In this section, we will use $\mathrm{W} 7-\mathrm{X}$ as a case study in how magnetic field arise in a stellarator.

The design and construction of any stellarator begins with the design of magnetic fields and the coils which give rise to them. The magnetic field of W7-X, which confines the plasma and gives it its shape, arise from a set of five unique three dimensional magnetic coil shapes. These coils are mirrored about five field periods resulting in 50 superconducting coils total. Two unique superconducting planar coils (again mirrored and repeated), allow fine tuning of the magnetic field rotational transform, along with radial repositioning of the magnetic flux surfaces. Figure 1 depicts the seven types of coils. These coils are supported on the inboard side of the device by a structural support ring. Sliding supports on the outboard side of the device allow some motion of the coils due to electromagnetic forces and reduce stresses in critical areas of the coil support structure. A small set of ten copper in-vessel 'control' coils allow tuning of the edge island chain, while five large trim coils provide a means to compensate critical error field components [24].

The superconducting coils could not be manufactured with infinite precision. Measurements of the coils upon delivery indicated deviations in the coil geometry by up to $3 \mathrm{~mm}$ for the non-planar coils and $5 \mathrm{~mm}$ for the planar coils. An extensive set of 


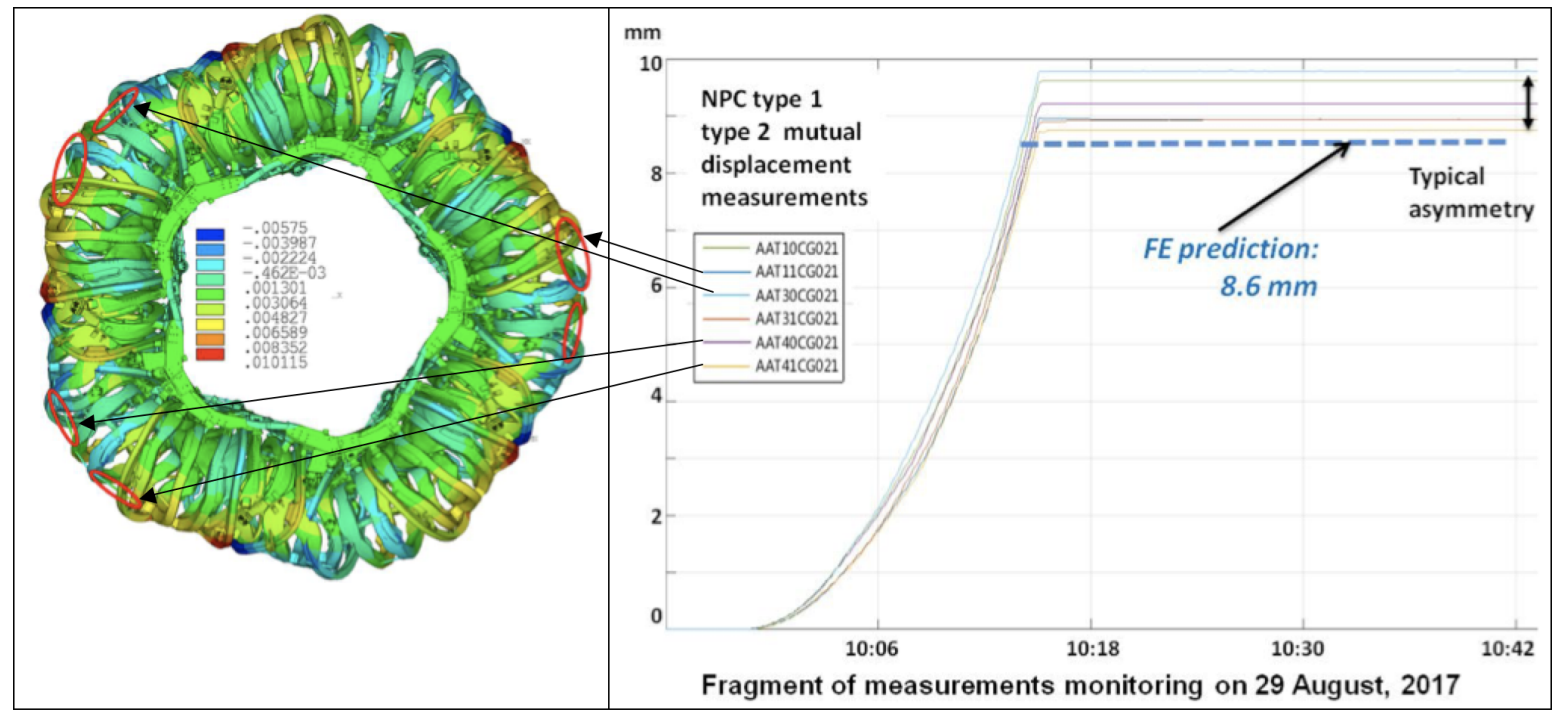

Figure 2. Location of distance measurement units indicated on deformed finite element (FE) model $(\mathrm{mm})$ by red ovals (left). Measurement example (high iota configuration with $2.5 \mathrm{~T}$ magnetic flux on plasma axis) in comparison with $\mathrm{FE}$ prediction (right).

measurements were used to predict the amplitude of the symmetry breaking error fields [22]. Complimentary computations were performed to estimate the optimum module position [25]. These computations allowed for optimum placement of each module, significantly reducing the total magnetic field error.

The interaction of the electromagnetic coils when energized result in deformation of the coils themselves. The 'self-force' of an energized three dimensional coil deforms the coil towards that of a simple planar circular hoop (this is the energetically favorable situation). This means that even if the errors in the construction and positioning of a coil do not exist, the rotational transform generated by a coil will be reduced as the coil is energized, as observed on W7-AS [6]. Again, assuming no errors in position exist, the coil-coil interactions will deform the positioning of the coils. This effect produces error fields which can deform the magnetic flux surfaces and produce high-order island chains. It is important to note that stellarator symmetry and field periodicity would not be broken in this case. It should be noted that while the calculation of forces from interacting coils is a linear calculation, inclusion of deformation results in a nonlinearity in the problem. Once coil manufacture defects and module re-alignment are included, the error fields become a configuration dependent quantity. Modeling said system becomes difficult and the problem involves calculation of electromagnetic forces and structural response in a cryogenically cooled high-vacuum system.

The measurement of coil deformation becomes key in predicting error fields, and stellarators provide a unique means to allow direct assessment of error fields. To verify coil deformation and provide a device safety metric, a set of strain gauges and distance measurement units have been installed on the coil cases of the W7-X coil set (figure 2) 
[26]. These diagnostics provide useful data for the verification of finite element model (FEM) predictions. However, the most useful diagnostic tool available to stellarators is the ability to directly image the magnetic field itself using a technique know as flux surface mapping [27]. This technique takes advantage of the fact that the confining field of a stellarator arises predominately from the magnetic coils, so that even without a plasma the magnetic flux surfaces are present. An electron beam can then be injected onto a flux surface and the electrons will stay tied to the field-lines on which they are injected. If a fluorescent mesh or swept rod (in the case of W7-X) are placed in the path of said electron beam, the flux surface can be imaged (producing a Poincaré like pattern). Additionally, neutral gas can be injected into the vessel and the field-lines directly imaged. These techniques were used extensively to measure the presence of a $n / m=1 / 2$ error field in W7-X [28]. Additionally, plasma wall interactions were studied with a technique known as a compass scan. Here, infrared cameras viewing all 10 divertor modules were used to assess the asymmetry in divertor heat load.

Additional sources of error fields can arise from auxiliary magnetic systems and materials that become magnetized. As previously mentioned, W7-X is equipped with two perturbative coil sets (one inside the plasma vessel, and one outside the cryostat). These coils allow both the introduction, and compensation of, error fields in a controlled manner. However, other sources of magnetic field exist in and around the W7-X device. These include the magnets in the neutral beam system, and possible magnetization of the ICRH antenna. These systems should also be considered when attempting to model the error fields of the W7-X device.

\section{Prediciton and measurement of error fields}

In this section, the effort to predict, assess, and correct the effects of error fields is presented. It should be noted that the last subsection examines the plasma-wall interactions and as such cannot directly disentangle error fields from misalignments of plasma facing components. However, combined with flux surface measurements this second effect can be evaluated. Ultimately, the primary goal of error field correction is to symeterize the divertor heat loads, so as to maximize power exhaust. This in turn maximizes the achievable plasma performance.

\subsection{Model Formulation}

Formulation of a predictive model for W7-X error field effects requires the development of a detailed coil model. This model must not only include the position of each coil as measured but must take into account the effect of cryogenic cool-down, deformation under electromagnetic forces, and the proper material parameters of the coil. In particular the proper Young's modulus must be specified for the coil system. One way to assess this is to compare the predicted change in plasma rotational transform with that measured in the device. 

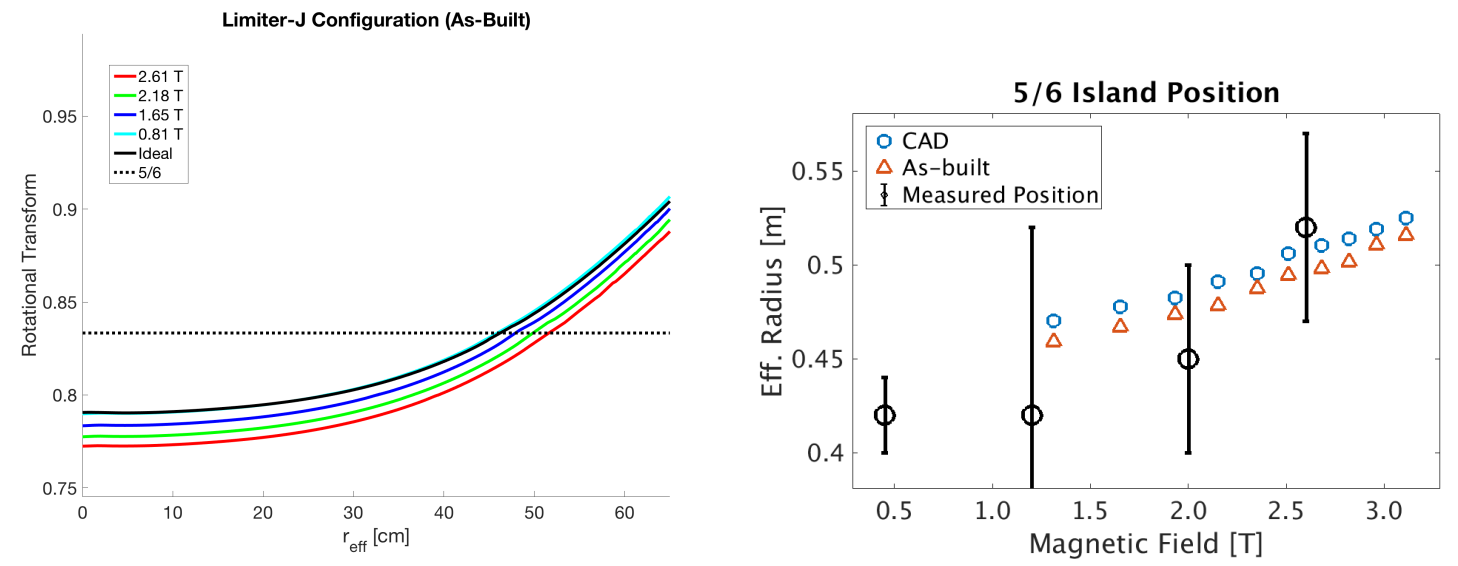

Figure 3. Modeling of the 'limiter' magnetic configuration shows a ridged decrease in the entire rotational transform profile (left). The position of the $5 / 6$ island is shown to be both a function of coil misalignment and electromagnetic load (right). The measured position of the $5 / 6$ island chain (from flux surface mapping) is shown.

The first experimental campaign (OP1.1) was conducted with an inboard limiter and a limiter magnetic configuration. This configuration had a $n / m=5 / 6$ island chain just inside the limiter itself, allowing it to be imaged. This was done at multiple magnetic field strengths and the shift of this island chain (in a radial sense) qualitatively agreed with modeling. That is, the chain moved radially outward as the field strength increased, thus confirming that the rotational transform was decreasing with increasing field strength. This is the coil flattening effect discussed in section 2 .

Simulations of coil deformation were then fed into the FIELDLINES code which allowed modeling of the flux surface mapping images. Figure 3 depicts the disagreements between modeling and flux surface measurements. Errors in island width measurements stem from difficulties imaging the islands at times and complications of mapping the islands to a sensible effective radial position. A refinement of the ANSYS magnetic system model was then introduced which improved the fit between model and experiment [29].

\subsection{Direct measurement of magnetic geometry}

The flux surface mapping technique allows stellarators to directly image the effect of error fields on both the magnetic geometry and topology of flux surfaces. Mapping of the 'standard' and 'high-iota' magnetic configurations provide initial estimates of the impact of error fields on the W7-X experiment. The 'high-iota' configuration was investigated with respect to the ability to correct error fields. This resulted in the demonstration of the correction of the $1 / 1$ error field component with the trim coils [30].

The main magnetic configuration of W7-X possess a $n / m=5 / 5$ island chain at its edge. In the magnetic fields arising from the ideal coil set, fields with toroidal mode number less than $n=5$ are not present and thus do not resonate with this island chain. 


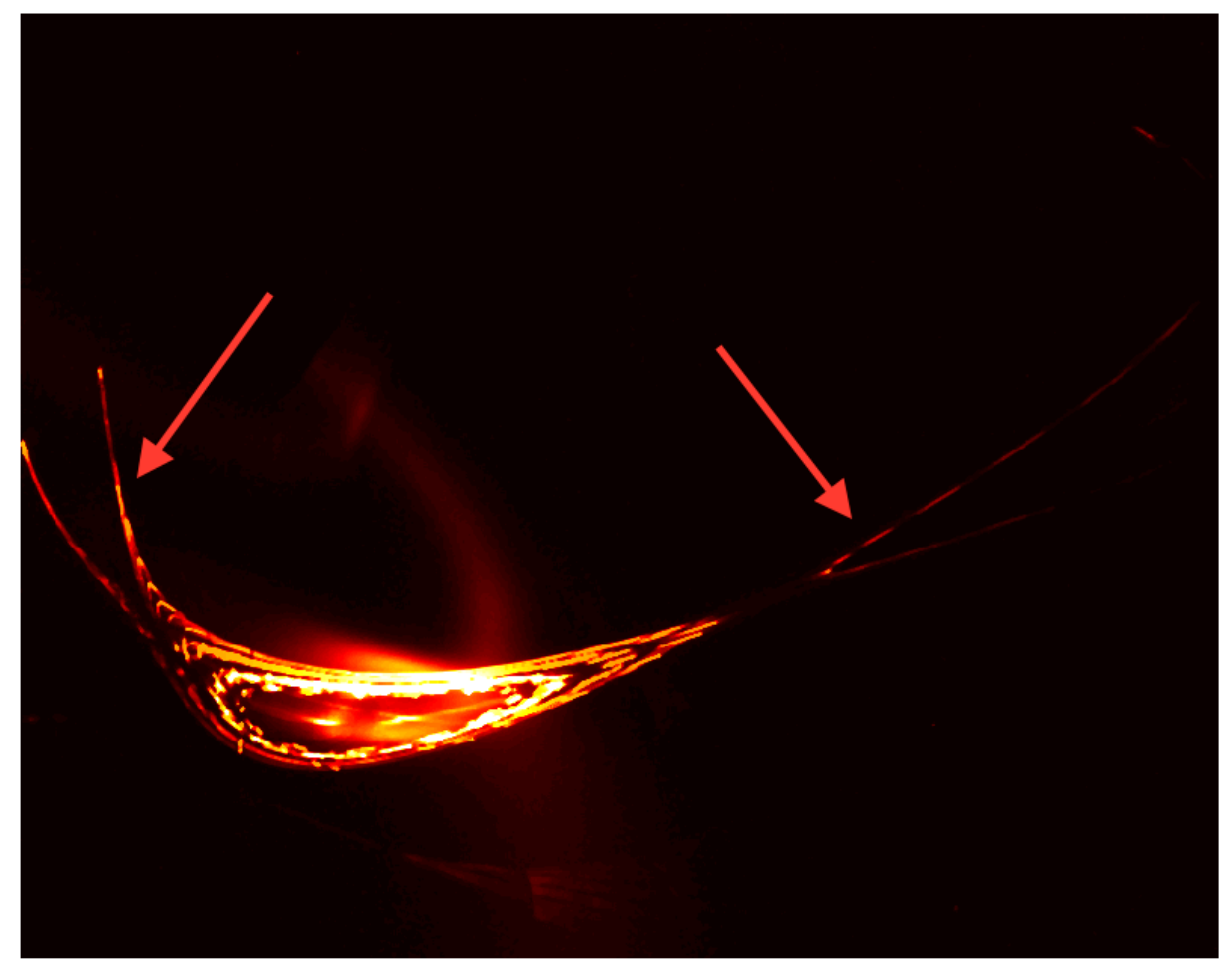

Figure 4. Flux surface mapping of the $5 / 5$ island chain showing the presence of $1 / 1$ features (arrows). If no $1 / 1$ error were present only the single island image would mapped and the bounding flux surfaces would not be connected.

However, previously mentioned 'as-built' geometry of the coils give rise to these lower order, non-field periodic, resonances. Flux surface mappings of the 'narrow mirror' configurations provided the first clear evidence of the interaction of a $1 / 1$ error field and the island divertor. In this magnetic configuration, the planar superconducting coils were used to reduce toroidal flux, increasing iota. This in turn moved the $5 / 5$ island chain off the divertor (radially inward) so it could be imaged. Figure 4 is a composite of images taken of the island flux surfaces in this configuration. The surfaces inside the 5/5 island chain are clearly visible. They only appear in one island as in this configuration each lobe of the $5 / 5$ island chain is a separate flux tube. The presence of surfaces outside the magnetic island lobe are attributed to the presence of a 1/1 error field. Flux surfaces inside and outside the island chain would not form a local x-point like structure as seen near the right arrow. Sharp truncations in the flux surfaces are due to the finite swing of the fluorescent rod. This provides clear evidence of error fields modifying the $5 / 5$ island chain.

The presence of the 1/1 error field was also measured in the 'high-iota' configuration [30]. In this configuration the rotational transform profile is modified (with the planar 


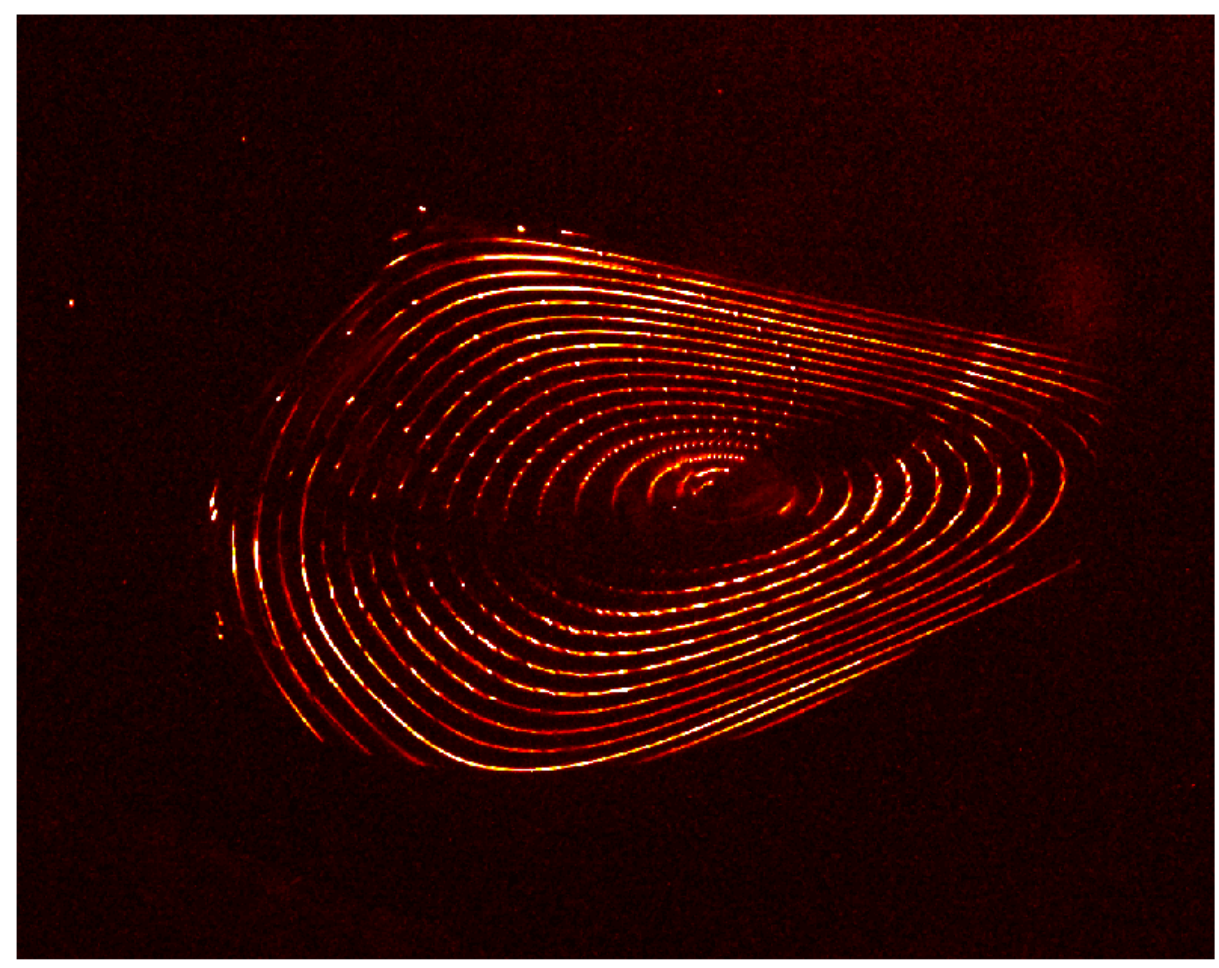

Figure 5. Flux surface mapping of the 'high-iota' configuration showing a clear shift in the axis position.

coils) so that the edge island chain is now a $n / m=5 / 4$ chain and is no longer resonant with the low order toroidal modes. However, this has the consequence of placing the rotational transform slightly above $\iota=1$ on axis. The presence of error fields which are resonant create a helical shift in the magnetic axis, so long as the rotational transform profile remains above one. Should the profile fall below one then a $1 / 1$ island chain should appear. We can directly assess the amplitude of the $n=1$ magnetic field as the amplitude of the magnetic field harmonics fall off as the distance from the magnetic axis to the $m$ power (where $m$ is the poloidal mode number). So in this configuration only the $m=0$ and $m=1$ harmonics should dominate. This isolates the $n / m=1 / 1$ component of the magnetic field.

Figure 5 clearly indicates the presence of a helical axis shift of approximately $10 \mathrm{~cm}$. While the measurement could be improved, the lack of a $m / n=1 / 1$ island chain clearly implies that the rotational transform is sufficiently above unity, but not so far above that the helical axis displacement disappears. While we can determine the physical amplitude of the displacement, we cannot directly assess the amplitude of the error field. This is because the amplitude of the displacement depends both on the amplitude of the error 


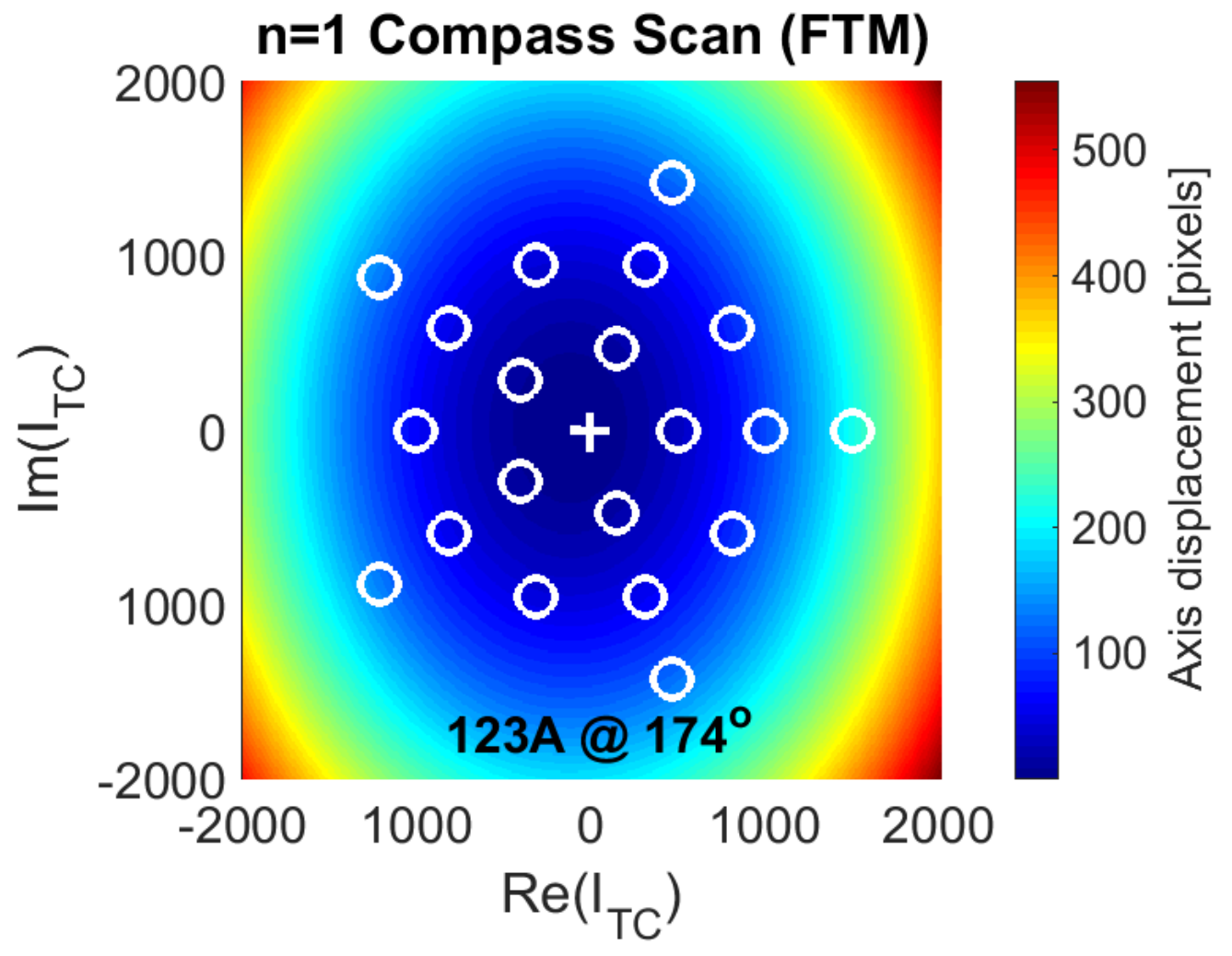

Figure 6. Analysis of compass scan data from flux surface mapping of the 'highiota' configuration in W7-X. Here each datapoint corresponds to a different phase and amplitude of a perturbing trim coil $n=1$ magnetic field. At each datapoint, the displacement of a axis from images is determined. Color contours represent a functional fit to the dataset indicating that for a trim coil setting of $123 \mathrm{~A}$ at a phase of $174^{\circ}$ the axis displacement is minimized.

field and the rotational transform of the configuration. As demonstrated before, the displacements which give rise to the error fields also give rise to changes in the rotational transform. A scan of field strength does show a dependence of the displacement on field strength but again the amplitude of the error field is also dependent on this quantity. Thus directly assessing the amplitude of the error field from this single measurement is not possible.

To solve this difficulty a compass scan was performed where the phase and amplitude of a probing magnetic field (due to the trim coil system) were varied and the change in axis displacement measured. Figure 6 depicts the analysis of this compass scan where the function $f=e+(x-c) / a+(y-d) / c$ is fit to the dataset (here $a, b, c, d$, and $e$ are coefficient to be determined through fitting). Here the amplitude and phase of the trim coils are defined by the function $I_{k}=A_{0} \times \cos (2 \pi(k-1) / 5-\phi)$, where $I_{k}$ is the current in the k'th trim coil, $A_{0}$ is the maximum coil current in the coils, and $\phi$ is the phase angle of the perturbation relative the module 1 trim coil centerline. The resulting fit suggests that for a trim coil setting of $A_{0}=123 A$ at $\phi=174^{\circ}$ the displacement in 

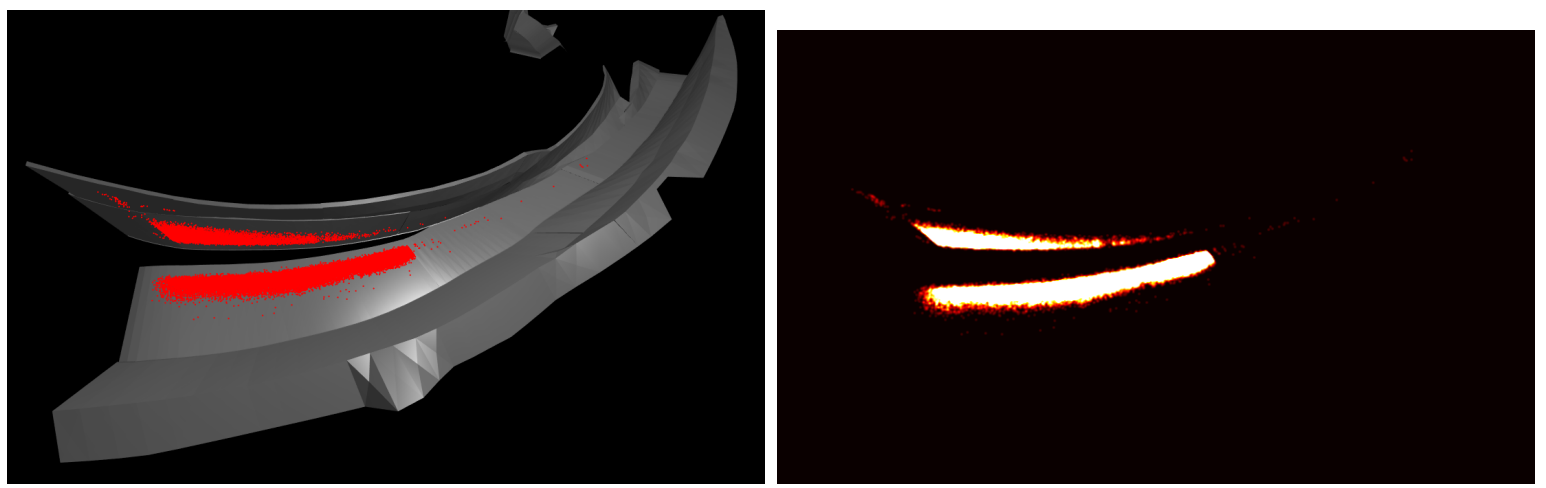

Figure 7. Particle diffusion simulation of the W7-X 'standard' configuration using the ideal coil. Red points indicate intersections of particle trajectories with the triangulated divertor and baffle surfaces (left). Colormap depicts a synthetic camera image of said data by binning of the strike points in the camera pixel geometry (right). Image geometry approximates the view of an actual IR camera.

the axis should be minimized. This compares well with FEM analysis of the 'as-built' coil set, where a trim coil setting of $A_{0}=134 A$ at $\phi=180^{\circ}$ is expected to minimize the error field. Application of these trim coil settings resulted in a shift in the axis position towards that estimated with field line tracing with the ideal coil set.

\subsection{Divertor operation}

Obtaining symmetry of divertor heat loads is the primary goal of error field correction in W7-X. One method to estimate divertor loads is to examine the effect of diffusing field line tracers given the different coil geometries. In this work the FIELDLINES code was applied using various coil models to estimate the level of asymmetry. In the experiment both infrared camera data and thermocouples located behind each divertor plate were used to measure the level of asymmetry. The trim coils were then used to perform compass scans in an attempt to find the magnetic field which provided the best divertor symmetry. The effect of an $n=0$ perturbation to the field were also explored.

The tracing of magnetic field lines with small diffusive kicks provides a quick and accurate tool for assessing the effect of error fields on divertor symmetry. Figure 7 depicts such as modeling effort using the ideal W7-X coil set and the 'standard' magnetic configuration of W7-X. In this simulation, $\sim 256000$ points are chosen on a flux surface found from vacuum field line tracing. Simulations are preformed by pushing the particles in both parallel and anti-parallel to the magnetic field with the inclusion of small kicks (see Appendix A for details of the diffusion model). Once a particle trajectory intersects the triangulated mesh of the divertor structure, the trajectory is stopped and the collision position recorded. Here an optimized version of the Möller and Trumbore method is utilized [31]. These points may be easily binned on the triangulated mesh. This is comparable to camera data only if the underlying mesh has a rather fine grid size. To avoid this we have mapped each strike point into the pixel space of our 

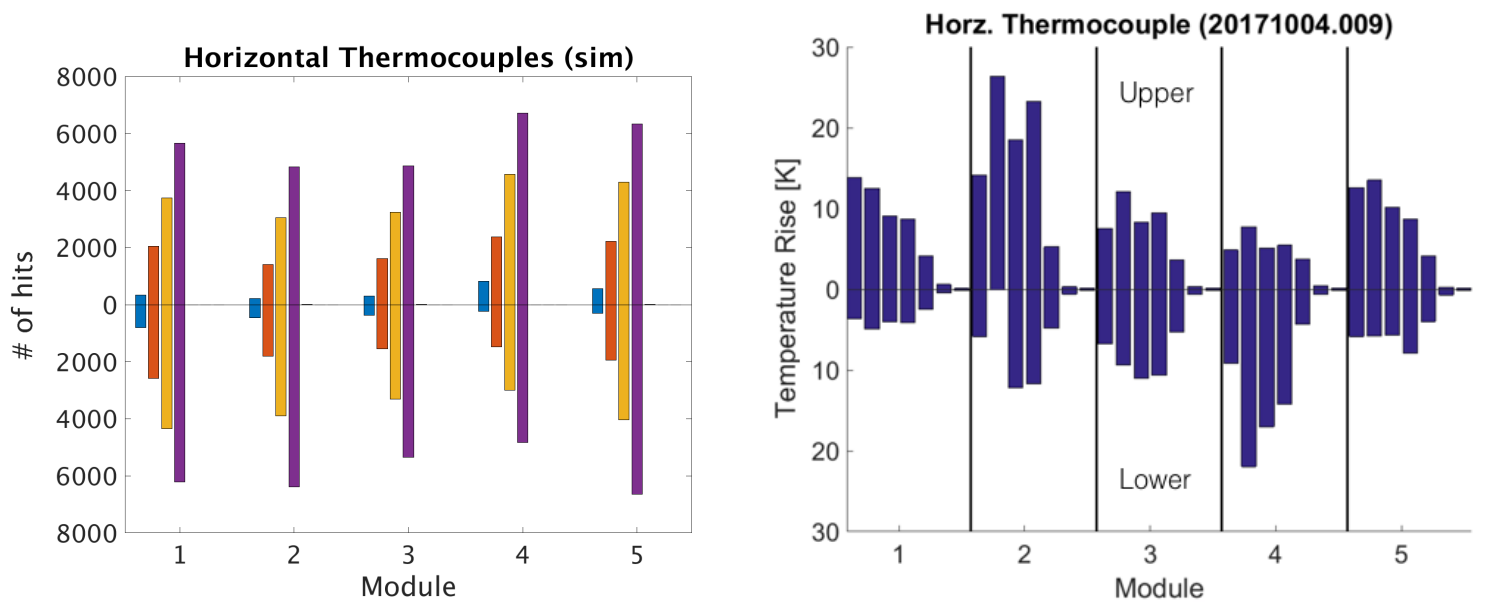

Figure 8. Analysis of the effect of error fields on divertor load symmetry based on simulation data (left) and measured data from experiments (right) for the 'standard' magnetic configuration. Each bar represents the temperature rise as measured by a thermocouple located in the main divertor (values in the upper half of the plot belong to upper divertors while those in the bottom to the lower divertors). Simulation data is computed by binning the number of diffusing particles hits on a given divertor finger. Both simulation and experiment appear to show consistency between which upper and lower modules receive the largest heat loads.

simulated camera. This decouples the resolution of the computational mesh from that of the simulated image. There was less than $1 \%$ variation in the particle strikes when comparing each divertor module for this ideal case.

Simulations were preformed using the electromagnetically loaded 'as-built' coil set to predict the level of divertor load asymmetry. Figure 8 shows the results of this simulation binned by divertor module and compared to experimental data (a proxy for the thermocouple divertor temperature rise). A small asymmetry is present in the simulation results when comparing the number of particles striking various divertor modules. Experimental data is taken from a $3 M W, 2 s$ Helium fueled discharge with no error field correction applied. Thermocouple temperature rise is calculated by a difference between the temperature before the discharge and the maximum temperature rise and the following $120 \mathrm{~s}$. Simulation data would suggest that the module 4 upper divertor would receive the largest heat load which is in clear conflict with the measured data. Experimental data indicates that the module 2 upper divertor receives the largest load. This suggest future refinements of the error field model. In particular, it should be noted that our model fails to account for up-down asymmetries due to particle drifts.

Infrared data for this discharge corroborates the thermocouple data. Figure 9 shows temperature data at the end of the discharge for 9 of the 10 divertor modules (note different lef to right ordering of modules as compared to figure 8). In these images the colors have been scaled to a common colormap for comparison. Here the upper divertor in module 2 and the lower divertor in module 4 are clearly experiencing the largest temperature rises. However, features in the IR camera data are present which were not 


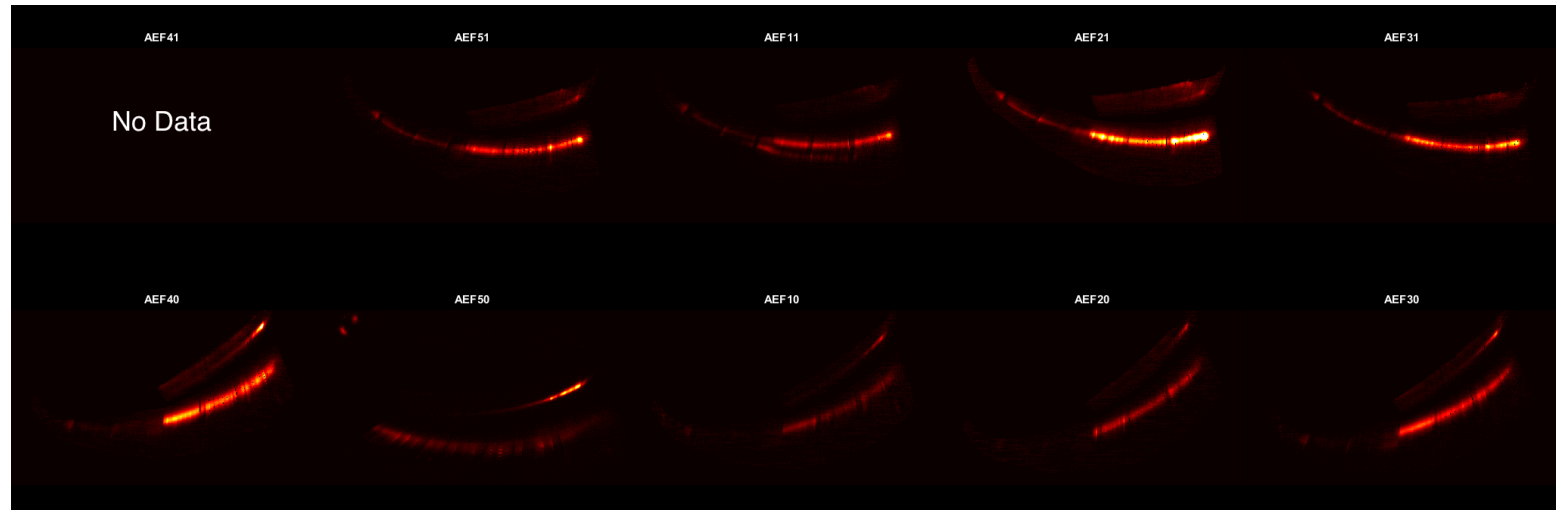

Figure 9. Infrared camera images for nine of the ten divertor modules in W7-X. Image data has been scaled to a common temperature map and data comes from near the end of the discharge. Non-divertor related regions of the camera data have been masked for clarity. Cameras named AEFX0 are located at the top of the machine observing the lower divertor while those labeled AEFX1 are located at the bottom observing the upper divertors.
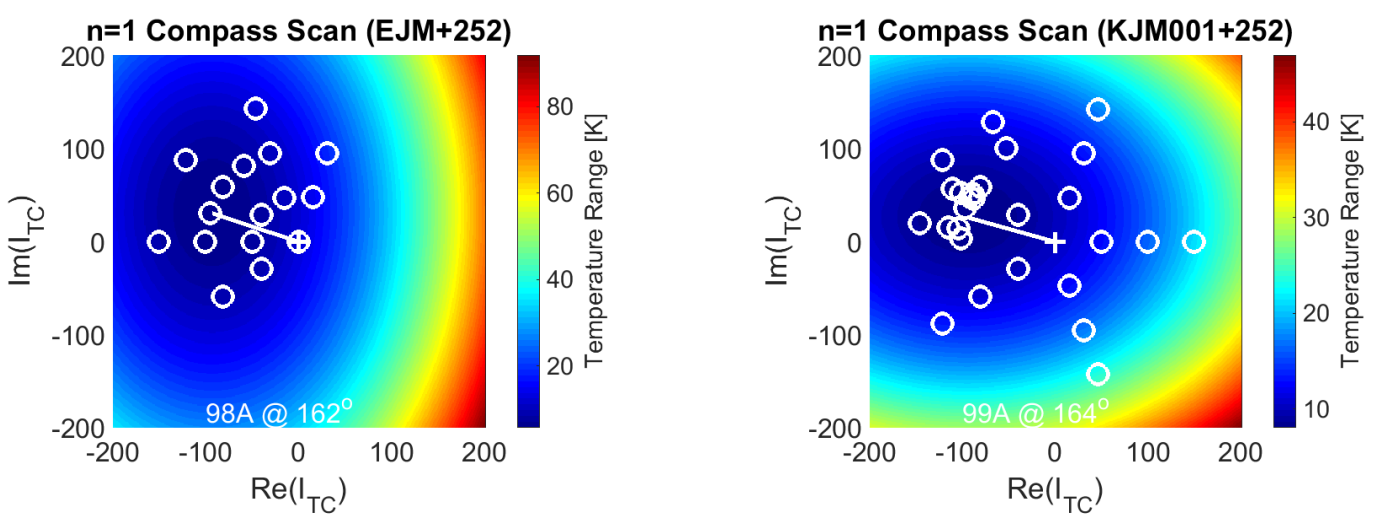

Figure 10. Compass scans ( $n=1)$ of the 'standard' (left) and 'narrow-mirror' (right) magnetic configurations showing a slight variation in compensating fields. Data is based on thermocouple measurements made at the backside of the divertor targets. Circles indicate data points and color contours are a best fit to that data. Text indicates the minimum in the fitting function.

present in the simulation data. The AEF11 camera (observing upper divertor in module 1) appears to show a double strike-line feature on the horizontal divertor target. This was not seen in the simulation data. Also some cameras show toroidally extended strike lines on the horizontal divertor while others show an abrupt discontinuity in the divertor strike line. This may be initial evidence of divertor mis-alignment. It should be noted that here we simply see a consistency between divertor temperature rise as measured by thermocouples and IR camera data. A more detailed analysis (at a later date) will generate heat flux maps from this dataset.

Compass scans $(n=1)$ were performed for two configurations with edge $5 / 5$ island divertor structures (figure 10). Both configurations exhibited similar amplitude 
correcting fields. Both configurations made use of approximately $-700 A$ of planar coil current to compensate the reduction of rotational transform. The configurations differed in that the 'narrow-mirror' configuration required variation in the non-planar coil currents while the 'standard' configuration utilized the same current in each nonplanar coil. This suggests that differences in electromagnetic loads between these configurations is small. It is worth noting that correcting field for these configurations and the 'high-iota' configuration differ in phase and amplitude. The differences arise from the large currents in the planar coils for the 'high-iota' configuration, as compared to the 'standard' and 'narrow mirror' configurations. Similar experiments performed with the limiter plasma suggested a slightly different phase for limiter heat flux balancing $[32,33]$. In this configuration the planar coils were energized with opposite sign and half the amplitude of the 'high-iota' configuration. This lends some credibility to the notion that the interaction between the planar and non-planar coils can significantly change the phase of the correcting error field. Although it should be noted that in these experiments, the effect of divertor mis-alignment cannot be disentangled.

When error field compensation is applied the symmetry of the divertor module heat load becomes greatly improved. The maximum temperatures rise in any horizontal divertor thermocouple is reduced as compared to the uncorrected temperature rise (figure 11). In terms or relative numbers the largest temperature discrepancy falls from a factor of almost 4 to values below 2 . The residual variance in temperatures appears to be due to a small uncompensated $n=2$ error field. Attempts to compensate these fields with the trim coils proved un-successful as the trim coils drive a similar amplitude $n / m=3 / 3$ when phased for $n=2$ correcting field. Future experiments will attempt to use the 10 in-vessel control coils to compensate the $n=2$ component.

A limited set of measurements were performed with the 'standard' configuration in a reversed field configuration. Assuming the error fields arise from the magnetic coils themselves, one would expect the symmetrizing field to be flipped in sign as well. Measurements could not confirm this. Both the previously established trim coil settings and those with flipped current (equivalently $-180^{\circ}$ phasing) failed to improve the symmetry of the divertor in the reversed field configuration. It was determined that the symmetrizing field lay somewhere in the range of $-90^{\circ}$ to $-180^{\circ}$ suggesting a complex interaction between the 'as-built' geometry and coil motion under electromagnetic forces. Experimental time was limited in this period of the campaign, so further investigation of this behavior is left to future work.

Scans of the effect of $n=0$ correction were also performed. In these scans, the trim coils were all set to the same current (and sign). This provided a uniform radial field, shifting the vacuum flux surface in the bean shaped cross section up and down (dependent on sign of the current). These experiments indicated temperature rises and decreases consistent with a vertical shift of the plasma. However, there was no appreciable change in divertor heat load symmetry. However, these experiments were preliminary without $n=1$ correction. Such experiments should be repeated with $n=1$ correction. 


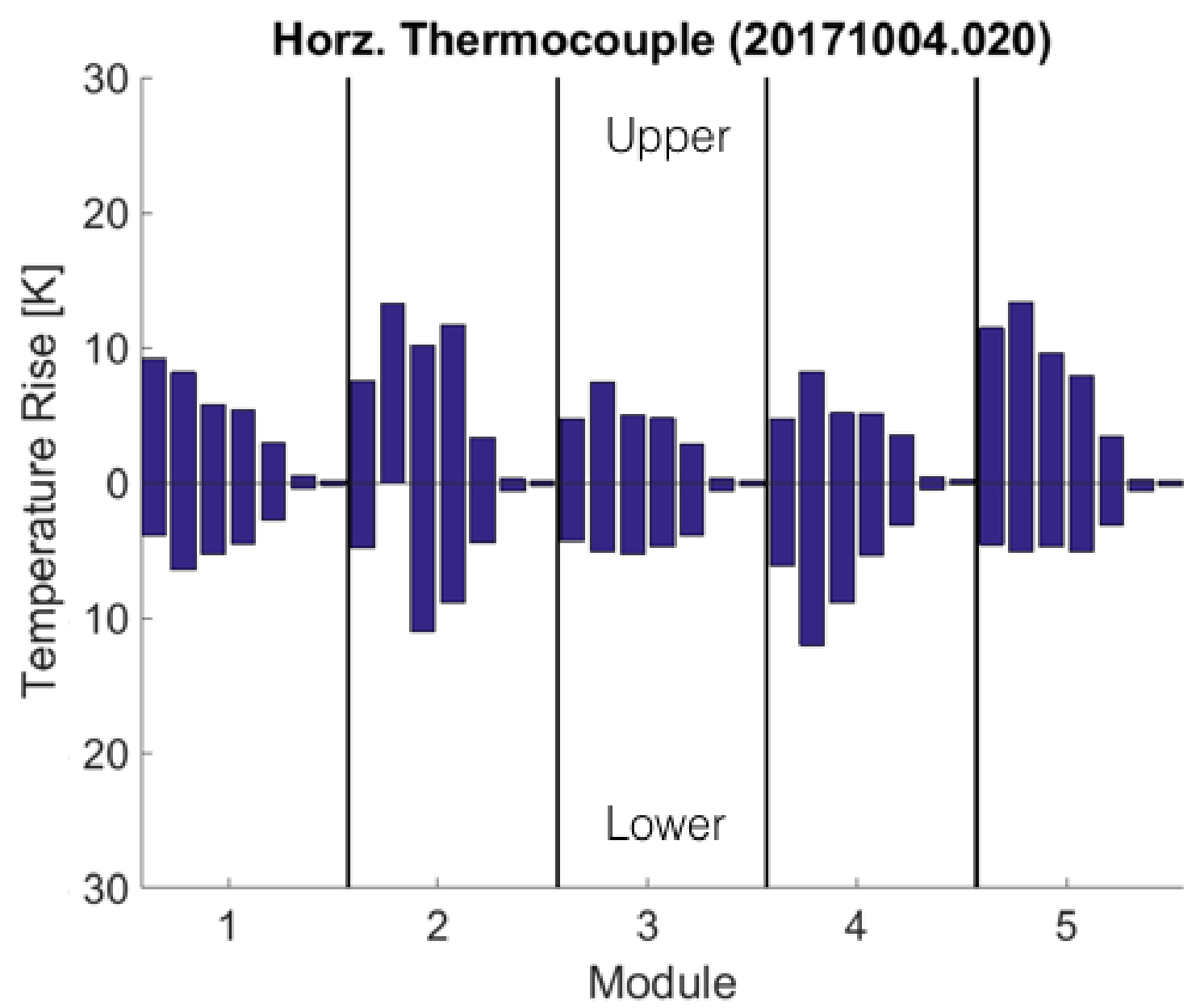

Figure 11. Horizontal divertor thermocouple temperature rise for symmetrizing trim coil currents in the 'standard' configuration. The temperature rise in module 2 and 4 are clearly much smaller than the uncorrected case (8). Each bar represents the temperature rise as measured by a thermocouple located in the main divertor (values in the upper half of the plot belong to upper divertors while those in the bottom to the lower divertors).

\section{Discussion}

The analysis of experimental data presented in this work confirms previously published results that the W7-X device was engineered and built to high accuracy. Such accuracy results in error field amplitudes which are small and well within the correcting ability of the trim and sweep coils. In practice, only approximately $5 \%$ the total trim coil correcting capabilities were utilized to provide significant improvements to divertor symmetry. The trim coil settings which provided improved symmetry in the device also appear to be configuration dependent. Specifically, the peak maximum trim coil current appears relatively fixed around $100 \mathrm{~A}$, but the phase appears to change as a function of the superconducting planar coil interaction. An overview of symmetrizing fields can be found in table 1 . The reversed field 'standard' configuration was omitted 
from this table as results could only conclude that flipping the sign of the correcting field was not sufficient to achieve symmetry.

Table 1. Trim coil configurations providing greatest divertor symmetry. Toroidal angle $\phi_{\text {Trim }}$ measured from the module 1 trim coil in a counter-clockwise fashion as viewed from above. We should note that the limiter configuration had a significantly reduced diagnostic set so the amplitude and phase which provided symmetry is difficult to discriminate.

\begin{tabular}{|c|c|c|c|} 
Configuration & $I_{A / B} A$ & $I_{\text {Trim }} A$ & $\phi_{\text {Trim }}{ }^{\circ}$ \\
\hline Standard & -700 & 98 & 162 \\
Narrow Mirror & -750 & 99 & 163 \\
High-Iota & -9790 & 134 & 180 \\
Limiter & 4980 & $\leq 200$ & $\sim-72$
\end{tabular}

Investigation into the error fields on W7-X have shown that a high level of manufacture precision was achieved in the construction of the device. One can now ask what can be learned from this data to help guide future stellarator designs. First, it should be pointed out that not all stellarators (or configurations of a given stellarator) are susceptible to the same magnetic error fields. So what holds for one stellarator may not necessarily hold for another. The key is the rotational transform profile and which rational numbers the profile crosses. If we examine the density of low order rationals we see that for rotational transform values below unity, the low order rationals tend to pile up below $\iota=1 / 2$. Thus keeping the entire rotational transform profile above this value significantly enhances the robustness to low order error fields. Second, it is also a well known fact that island widths tend to scale as the shear in the rotational transform. High shear suppresses the formation of islands at rational surfaces. However, both these facts are well established, and often difficult to incorporate into a design.

What we have demonstrated in this work is that trim coils can remedy the effects of error fields. This fact has been well established in tokamaks for decades. The assessment and correction of error fields from sources not directly part of the coil system (breeder blankets in reactor designs for example) is a straightforward process. For a component generating a given 'erroneous' magnetic field, a trim coil set capable of mitigation is required. However, only recently have tools been developed which can directly assess the relaxation in tolerances for coil construction [34]. Such codes are already providing reliable estimates the most sensitive coil deformations [15]. As a future work it is proposed that a stellarator coil set be modeled in said code including a set of trim coils. One should then be able to gauge the decrease in sensitivity to coil deformations. Said work may also identify key trim coil shapes which reduce coil alignment tolerances.

What was not addressed in this work were the impacts of divertor displacements, plasma response modeling, or particle drifts. The compass scans performed in the 'standard' and 'narrow mirror' configurations would nominally correct both the intrinsic error field and the effect of divertor mis-alignment. Thus it is difficult to disentangle 
the two effects. One may reason that the discrepancy between the error field corrections of these configurations and the high-iota is exactly due to this effect. However, this overlooks the fact that, in the high iota,the resonance of interest is located farther toward the plasma core and thus requires a slightly larger drive to compensate. Additionally, the error field should arise from the coil set itself while the correcting field arrises from the trim coils located farther away from the resonance. In the future, a 'low-iota' configuration will be run with the $n / m=5 / 6$ island chain at it's edge and no $\iota=1$ surface in the plasma. Analysis of this configuration should provide clarity on divertor alignment issues. Moreover, by reversing the magnetic field of this configuration, one could assess the role of divertor drifts on the up-down divertor asymmetry. One could also use the trim coils and 'high-iota' configuration to attempt to run a plasma discharge with large helical axis shift, this would then quantify the effect of surface distortions on plasma performance.

\section{Acknowledgments}

The authors would like to thank N. Logan, C. Paz-Soldan, J. Geiger, G. Wurden, and J. Harris for their useful discussions. This work has been carried out within the framework of the EUROfusion Consortium and has received funding from the Euratom research and training programme 2014-2018 under grant agreement No 633053. The views and opinions expressed herein do not necessarily reflect those of the European Commission.

\section{Appendix A. Model for field line diffusion}

The FIELDLINES code is designed to push particles on a cylindrical grid $(R, \phi, Z)$. The equation for a following a magnetic field line is simply one for the the stream-lines of the magnetic field $\partial \vec{x} / \partial s=\vec{b}$, where $\vec{x}$ is the position of the particle along the field line, $\vec{b}=\vec{B} /|\vec{B}|$ is the normalized magnetic field, and $s$ is a parameter characterizing the path along the field line (sometimes referred to as a pseudo-time). In cylindrical coordinates, the dependance on $s$ can be eliminated and the equation reduced from three components to two, requiring one to solve

$$
\begin{aligned}
& \frac{\partial R}{\partial \phi}=R \frac{B_{R}}{B_{\phi}} \\
& \frac{\partial Z}{\partial \phi}=R \frac{B_{Z}}{B_{\phi}}
\end{aligned}
$$

where subscripts denote components of the magnetic field in cylindrical coordinates. To incorporate diffusion a modified equation must be solved, specifically $\partial \vec{x} / \partial s=\vec{b}+\vec{\delta}$

where $\vec{\delta}$ is a small random kick to simulate diffusion. This equation can then again be simplified into two equations in terms of the toroidal angle $(\phi)$

$$
\frac{\partial R}{\partial \phi}=R \frac{B_{R}}{B_{\phi}}+\frac{|B|}{B_{\phi}} \delta_{R}
$$



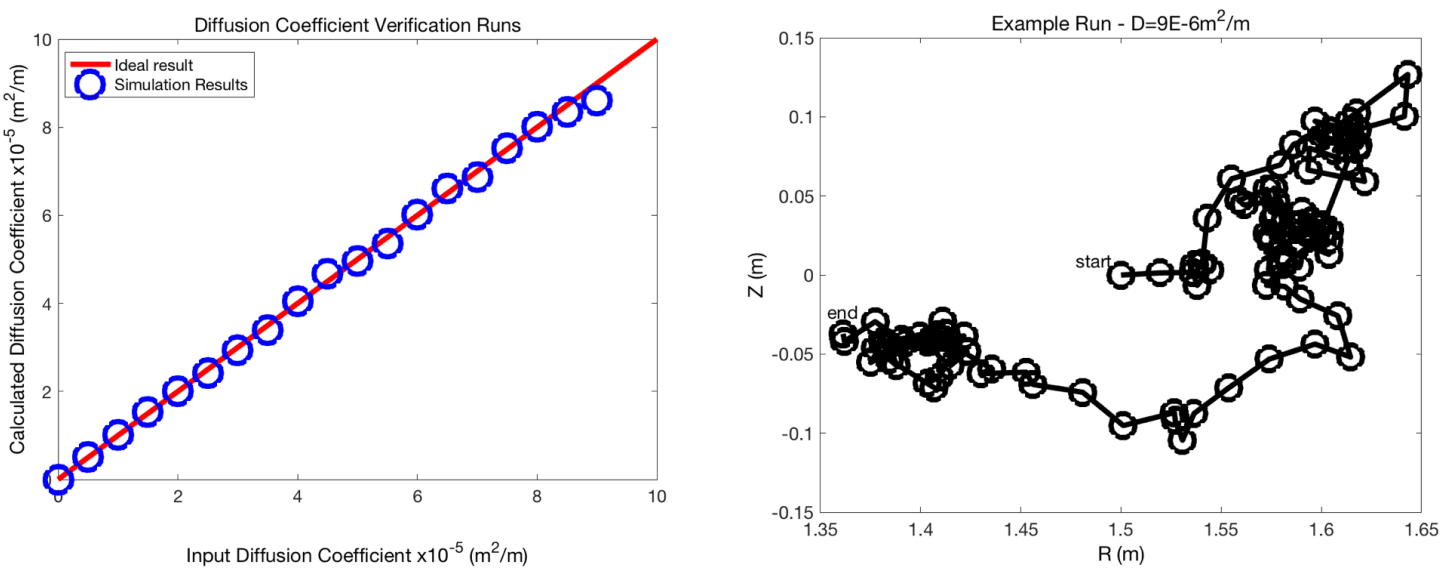

Figure A1. Test of diffusion coefficient as implemented in the FIELDLINES code. Good agreement is seen between input and measured value of the diffusion coefficients (left). Measured values are determined from the mean squared displacement of a particle after 100 toroidal transits. A depiction of the random walk pattern for one particle is shown for reference (right).

$$
\frac{\partial Z}{\partial \phi}=R \frac{B_{Z}}{B_{\phi}}+\frac{|B|}{B_{\phi}} \delta_{Z}
$$

where we've assumed the component of the diffusion in the toroidal direction $\delta_{\phi}$ can be safely ignored. In systems with predominantly toroidal field (tokamaks and stellarators) this is similar to the assumption of purely radial diffusion. Thus our diffusion operator becomes a displacement in the 2D RZ plane of $\frac{|B|}{B_{\phi}} \vec{\delta}$, applied at each regular interval in $\phi$.

The 2D Brownian motion of particles is characterized by the standard deviation of displacement over a signal timestep as $k=\sqrt{2 D d s}=\sqrt{2 D R|B| \Delta \phi / B_{\phi}}$. Here $D$ is the diffusion coefficient. Thus our diffusive terms can be implemented as

$$
\frac{|B|}{B_{\phi}} \delta_{R / Z}=\frac{|B|}{B_{\phi}} n_{R / Z} \sqrt{2 D R|B| \frac{\Delta \phi}{B_{\phi}}}
$$

where the subscripts refer to the $\mathrm{R}$ and $\mathrm{Z}$ components, and $n$ are normally distributed random values with $\sigma=1$.

Figure A1 depicts results for a validation activity of this operator in the FIELDLINES code. A system with purely toroidal field was constructed $\left(B_{R}=B_{Z}=0\right)$. For a given choice of the diffusion coefficient $D, 4096$ particles were followed for 100 toroidal transits. The mean squared displacement of these particles were then used to calculate an effective diffusion. Comparison with the input value of the diffusion coefficient shows excellent agreement.

[1] D W Kerst. The influence of errors on plasma-confining magnetic fields. Journal of Nuclear Energy. Part C, Plasma Physics, Accelerators, Thermonuclear Research, 4(4):253-262, December 2002. 
[2] R M Sinclair, J C Hosea, and G V Sheffield. MAGNETIC SURFACE MAPPINGS BY STORAGE OF PHASE-STABILIZED LOW-ENERGY ELECTRON BEAMS. Applied Physics Letters, 17(2):92-95, July 1970.

[3] R J Colchin, F S B Anderson, A C England, R F Gandy, J H Harris, M A Henderson, D L Hillis, R R Kindsfather, D K Lee, D L Million, M Murakami, George Hutch Neilson, M J Saltmarsh, and C M Simpson. Electron beam and magnetic field mapping techniques used to determine field errors in the ATF torsatron. Review of Scientific Instruments, 60(8):2680, 1989.

[4] R J Colchin, S I Fedotov, Jeffrey Harris, D K Lee, F I Ozherelev, O S Pavlichenko, D P Pogozhev, J A Rome, J D Treffert, and V M Zalkind. Magnetic Field Alignment Studies for the URAGAN3 Torsatron. Technical Report ORNL/TM-9685, Oak Ridge National Laboratory, Oak Ridge, February 1986.

[5] R Jaenicke, E Ascasíbar, P Grigull, I Lakicevic, A Weller, M Zippe, H Hailer, and K Schwörer. Detailed investigation of the vacuum magnetic surfaces on the W7-AS stellarator. Nuclear Fusion, 33(5):687-704, May 1993.

[6] Matthias Otte and R. Jaenicke. Magnetic flux surface measurements at Wendelstein W7-AS. Stellarator News, 100:2-5, September 2005.

[7] Matthias Otte, D Andruczyk, E Holzhauer, J Howard, R König, L Krupnik, H P Laqua, O Lischtschenko, S Marsen, and J Schacht. The WEGA stellarator: results and prospects. AIP Conference Proceedings, 993(3):3-10, 2008.

[8] J T Peterson, G J Hartwell, S F Knowlton, James D Hanson, R F Kelly, and C Montgomery. Initial Vacuum Magnetic Field Mapping in the Compact Toroidal Hybrid. Journal of Fusion Energy, 26(1-2):145-148, December 2006.

[9] Enrique Ascasibar, Jiang Qin, and Antonio López-Fraguas. Magnetic Surface Mapping Experiments in TJ-II. Journal of Plasma Fusion Research, 1:183-186, October 1997.

[10] George Hutch Neilson, C O Gruber, J H Harris, D J Rej, R T Simmons, and R L Strykowsky. Lessons learned in risk management on NCSX. In 2009 23rd IEEE/NPSS Symposium on Fusion Engineering, pages 1-6. IEEE, 2009.

[11] R L Strykowsky, Thomas Brown, J Chrzanowski, M Cole, P Heitzenroeder, George Hutch Neilson, Donald Rej, and M Viol. Engineering cost \& schedule lessons learned on NCSX. In 2009 23rd IEEE/NPSS Symposium on Fusion Engineering, pages 1-4. IEEE, 2009.

[12] Tomohiro Morisaki, Mamoru Shoji, Suguru Masuzaki, Satoru Sakakibara, Hiroshi Yamada, Akio Komori, and Osamu Motojima. Flux Surface Mapping in LHD. Fusion Science and Technology, 58(1):465-470, 2010.

[13] Santhosh T A Kumar, Boyd D Blackwell, and Jeffrey H Harris. Determination of error field sources by accurate mapping of the magnetic geometry of the H-1 heliac. Nuclear Fusion, 49(3):035001-12, February 2009.

[14] K C Hammond, A Anichowski, P W Brenner, Thomas Sunn Pedersen, S RAFTOPOUlOS, $\mathrm{P}$ Traverso, and F A Volpe. Experimental and numerical study of errorfields in the CNT stellarator. Plasma Physics and Controlled Fusion, 58(07):074002, May 2016.

[15] Caoxiang Zhu, Stuart R Hudson, Samuel A Lazerson, Yuntao Song, and Yuanxi Wan. Hessian matrix approach for determining error field sensitivity to coil deviations. submitted to Plasma Physics and Controlled Fusion, 2018.

[16] R J Buttery, M De Benedetti, David A Gates, Yu Gribov, T C Hender, R J La Haye, P Leahy, J A Leuer, A W Morris, A Santagiustina, J T Scoville, B J D Tubbing, JET Team, COMPASSD Research Team, and DIII-D Team. Error field mode studies on JET, COMPASS-D and DIII-D, and implications for ITER. Nuclear Fusion, 39(11Y):1827-1835, May 2002.

[17] Thomas Rummel, Konrad Riße, Gunnar Ehrke, Kerstin Rummel, Andre John, Thomas Mönnich, Klaus-Peter Buscher, Walter H Fietz, Reinhard Heller, Olaf Neubauer, and Anatoly Panin. The Superconducting Magnet System of the Stellarator Wendelstein 7-X. IEEE Transactions on Plasma Science, 40(3):769-776, March 2012.

[18] Thomas Rummel, Konrad Riße, Frank Fullenbach, Matthias Koppen, Johann Kisslinger, Tom 
Brown, Ron Hatcher, Stephen Langish, Mike Mardenfeld, and George Hutch Neilson. The Wendelstein 7-X Trim Coil System. IEEE Transactions on Applied Superconductivity, 24(3):14, 2014.

[19] Maciej Krychowiak, A Adnan, A Alonso, Tamara Andreeva, J Baldzuhn, T Barbui, M Beurskens, W Biel, C Biedermann, B D Blackwell, H S Bosch, Sergey A Bozhenkov, R Brakel, T Bräuer, B Brotas de Carvalho, R Burhenn, B Buttenschön, A Cappa, G Cseh, A Czarnecka, A Dinklage, P Drews, A Dzikowicka, F Effenberg, M Endler, V Erckmann, T Estrada, O Ford, T Fornal, H Frerichs, G Fuchert, Joachim Geiger, O Grulke, J H Harris, H-J Hartfuß, D Hartmann, D Hathiramani, M Hirsch, U Höfel, S Jabłonski, M W Jakubowski, J Kaczmarczyk, T Klinger, S Klose, J Knauer, G Kocsis, R König, P Kornejew, A Kramer-Flecken, N Krawczyk, T Kremeyer, I Ksiazek, M Kubkowska, A Langenberg, H P Laqua, M Laux, Samuel Lazerson, Y Liang, S C Liu, A Lorenz, A O Marchuk, S Marsen, V Moncada, D Naujoks, George Hutch Neilson, O Neubauer, U Neuner, H Niemann, J W Oosterbeek, Matthias Otte, N Pablant, E Pasch, Thomas Sunn Pedersen, F Pisano, K Rahbarnia, L Ryc, O Schmitz, S Schmuck, W Schneider, T Schröder, H Schuhmacher, B Schweer, B Standley, T Stange, L A Stephey, J Svensson, T Szabolics, T Szepesi, H Thomsen, J M Travere, H Trimino Mora, H Tsuchiya, G M Weir, U Wenzel, A Werner, B Wiegel, T Windisch, Robert C Wolf, G A Wurden, D Zhang, A Zimbal, S Zoletnik, and W7-X Team. Overview of diagnostic performance and results for the first operation phase in Wendelstein 7-X (invited). Review of Scientific Instruments, 87(11):11D304, October 2016.

[20] J Nührenberg and R Zille. Stable stellarators with medium $\beta$ and aspect ratio. Physics Letters A, 114(3):129-132, 1986.

[21] Sergey A Bozhenkov, Joachim Geiger, M Grahl, Johann Kisslinger, A Werner, and R C Wolf. Fusion Engineering and Design. Fusion Engineering and Design, 88(11):2997-3006, November 2013.

[22] Tamara Andreeva, T Bräuer, M Endler, Johann Kisslinger, and Y Igitkhanov. Analysis of the magnetic field perturbations during the assembly of Wendelstein 7-X. Fusion Science and Technology, 46(2):388-394, September 2004.

[23] Thomas Sunn Pedersen, Matthias Otte, Samuel Lazerson, Per Helander, Sergey A Bozhenkov, Christoph Biedermann, T Klinger, Robert C Wolf, and H S Bosch. Confirmation of the topology of the Wendelstein 7-X magnetic field to better than 1: 100,000. Nature Communications, 7, 2016.

[24] R Stadler, A Vorköper, J Boscary, A Cardella, F Hurd, Ch Li, B Mendelevitch, A Peacock, and $\mathrm{H}$ Pirsch. The in-vessel components of the experiment WENDELSTEIN 7-X. Fusion Engineering and Design, 84(2-6):305-308, June 2009.

[25] H S Bosch, Robert C Wolf, Tamara Andreeva, J Baldzuhn, D Birus, T Bluhm, T Bräuer, H Braune, Victor Bykov, A Cardella, F Durodié, M Endler, V Erckmann, G Gantenbein, D Hartmann, D Hathiramani, P Heimann, B Heinemann, C Hennig, M Hirsch, D Holtum, J Jagielski, J Jelonnek, W Kasparek, T Klinger, R König, P Kornejew, H Kroiss, J G Krom, G Kühner, H Laqua, H P Laqua, C Lechte, M Lewerentz, J Maier, P McNeely, A Messiaen, G Michel, J Ongena, A Peacock, Thomas Sunn Pedersen, R Riedl, H Riemann, P Rong, Norbert Rust, J Schacht, F Schauer, R Schroeder, B Schweer, Anett Spring, A Stabler, Y Turkin, L Wegener, A Werner, D Zhang, M Zilker, T Akijama, R Alzbutas, E Ascasíbar, M Balden, M Banduch, Ch Baylard, W Behr, Craig D Beidler, A Benndorf, T Bergmann, C Biedermann, B Bieg, W Biel, M Borchardt, G Borowitz, V Borsuk, Sergey A Bozhenkov, R Brakel, H Brand, Thomas Brown, B Brucker, R Burhenn, K P Buscher, C Caldwell-Nichols, A Cappa, A Carls, P Carvalho, Ł Ciupiński, M Cole, J Collienne, A Czarnecka, G Czymek, G Dammertz, C P Dhard, V I Davydenko, A Dinklage, M Drevlak, S Drotziger, A Dudek, P Dumortier, G Dundulis, P v Eeten, K Egorov, T Estrada, H Faugel, J Fellinger, Y Feng, H Fernandes, W H Fietz, W Figacz, F Fischer, J Fontdecaba, A Freund, T Funaba, H Fünfgelder, A Galkowski, David A Gates, L Giannone, J M García Regaña, Joachim Geiger, S Geißler, 
H Greuner, M Grahl, S Groß, A Grosman, H Grote, O Grulke, M Haas, L Haiduk, H-J Hartfuß, J H Harris, D Haus, B Hein, P Heitzenroeder, P Helander, R Heller, C Hidalgo, D Hildebrandt, H Höhnle, A Holtz, E Holzhauer, R Holzthüm, A Huber, H Hunger, F Hurd, M Ihrke, S Illy, A Ivanov, S Jablonski, N Jaksic, M W Jakubowski, R Jaspers, H Jensen, H Jenzsch, J Kacmarczyk, T Kaliatk, J Kallmeyer, U Kamionka, R Karaleviciu, S Kern, M Keunecke, R Kleiber, J Knauer, R Koch, G Kocsis, A Könies, M Köppen, R Koslowski, J Koshurinov, A Kramer-Flecken, R Krampitz, Y Kravtsov, Maciej Krychowiak, G Krzesinski, I Ksiazek, Fr Kubkowska, A Kus, S Langish, R Laube, M Laux, Samuel Lazerson, M Lennartz, C Li, R Lietzow, A Lohs, A Lorenz, F Louche, L Lubyako, A Lumsdaine, A Lyssoivan, Henning Maaßberg, P Marek, C Martens, N Marushchenko, M Mayer, B Mendelevitch, Ph Mertens, David R Mikkelsen, A Mishchenko, B Missal, T Mizuuchi, H Modrow, T Mönnich, T Morizaki, S Murakami, F Musielok, M Nagel, D Naujoks, George Hutch Neilson, O Neubauer, U Neuner, R Nocentini, J M Noterdaeme, Carolin Nührenberg, S Obermayer, G Offermanns, H Oosterbeek, Matthias Otte, A Panin, M Pap, S Paquay, E Pasch, X Peng, S Petrov, D Pilopp, H Pirsch, B Plaum, F Pompon, M Povilaitis, J Preinhaelter, O Prinz, F Purps, T Rajna, S Récsei, Allan H Reiman, D Reiter, J Remmel, S Renard, V Rhode, J Riemann, S Rimkevicius, K Riße, A Rodatos, I Rodin, M Romé, H J Roscher, K Rummel, Thomas Rummel, A M Runov, L Ryc, J Sachtleben, A Samartsev, M Sanchez, F Sano, A Scarabosio, M Schmid, H Schmitz, O Schmitz, M Schneider, W Schneider, L Scheibl, M Scholz, G Schröder, M Schröder, J Schruff, H Schumacher, I V Shikhovtsev, M Shoji, G Siegl, J Skodzik, M Smirnow, E Speth, Don A Spong, R Stadler, Z Sulek, V Szabó, T Szabolics, T Szetefi, Z Szökefalvi-Nagy, A Tereshchenko, H Thomsen, M Thumm, D Timmermann, H Tittes, K Toi, M Tournianski, U v Toussaint, J Tretter, S Tulipán, P Turba, R Uhlemann, J Urban, E Urbonavicius, P Urlings, S Valet, D Van Eester, M Van Schoor, M Vervier, H Viebke, R Vilbrandt, M Vrancken, and T and... Wauters. Technical challenges in the construction of the steady-state stellarator Wendelstein 7-X. Nuclear Fusion, 53(12):126001, November 2013.

[26] V. Bykov, K. Egorov, J. Fellinger, J. P. Kallmeyer, F. Schauer, and M. Gasparotto. Wendelstein 7-x mechanical instrumentation system for commissioning and operation. Fusion Science and Technology, 68(2):267-271, 2015.

[27] Matthias Otte, D Aßmus, C Biedermann, Sergey A Bozhenkov, T Bräuer, A Dudek, Joachim Geiger, G Kocsis, Samuel Lazerson, Thomas Sunn Pedersen, F Schauer, T Szepesi, B Standley, and the W7-X Team. Setup and initial results from the magnetic flux surface diagnostics at Wendelstein 7-X. Plasma Physics and Controlled Fusion, 58(06):064003, May 2016.

[28] Samuel Lazerson, Matthias Otte, Sergey A Bozhenkov, Christoph Biedermann, Thomas Sunn Pedersen, and the W7-X Team. First measurements of error fields on W7-X using flux surface mapping. Nuclear Fusion, 56(10):106005, August 2016.

[29] Victor Bykov, K Egorov, J Fellinger, J P Kallmeyer, F Schauer, and M Gasparotto. Wendelstein 7-X Mechanical Instrumentation System for Commissioning and Operation. Fusion Science and Technology, 68(2):267-271, March 2017.

[30] S.A. Bozhenkov, M. Otte, C. Biedermann, M. Jakubowski, S. A. Lazerson, T.S. Pedersen, R Wolf, and the W7-X Team. Measurements and correction of the 1/1 error field in wendelstein 7-x. submitted to Nuclear Fusion, XX(X):XX, 2018.

[31] Tomas Möller and Ben Trumbore. Fast, minimum storage ray-triangle intersection. Journal of Graphics Tools, 2(1):21-28, 1997.

[32] Samuel Lazerson, Matthias Otte, Marcin Jakubowski, Ben Israeli, Glen A Wurden, Uwe Wenzel, Tamara Andreeva, Sergey A Bozhenkov, Christoph Biedermann, Gabor Kocsis, Tamas Szepesi, Joachim Geiger, Thomas Sunn Pedersen, David Gates, and the W7-X Team. Error field measurement, correction and heat flux balancing on Wendelstein 7-X. Nuclear Fusion, 57(4):046026-9, March 2017.

[33] Sergey A Bozhenkov, M W Jakubowski, H Niemann, Samuel Lazerson, G A Wurden, C Biedermann, G Kocsis, R König, F Pisano, L A Stephey, T Szepesi, U Wenzel, Thomas Sunn 
Pedersen, Robert C Wolf, and W7-X Team. Effect of error field correction coils on W7-X limiter loads. Nuclear Fusion, 57(12):126030-11, September 2017.

[34] Caoxiang Zhu, Stuart R Hudson, Yuntao Song, and Yuanxi Wan. New method to design stellarator coils without the winding surface. Nuclear Fusion, 58(1):016008-13, November 2017. 